\title{
The effects of isometric exercise types on pain and muscle activity in patients with low back pain
}

\author{
Hyun-Seung Rhyu', Hun-Kyung Park'2, Jung-Sub Park' ${ }^{3}$ Hye-Sang Park,** \\ 'Department of Sports Health Medicine, College of Health Science, Jungwon University, Geosan-gun, Chungcheongbuk-do, Korea \\ ${ }^{2}$ Department of Physiology, Kyung Hee University College of Medicine, Seoul, Korea \\ ${ }^{3}$ Department of Physical Education, Korea Air Force Academy, Cheongju, Korea \\ ${ }^{4}$ Department of Sports Health Management, College of Physical Education, University of Suwon, Hwaseong, Korea
}

The purpose of the present study is to evaluate the effects of isometric exercise types on low back pain (LBP) patients. Isometric exercise types were mat exercise and I-Zer exercise. Subjects were divided into 3 groups: LBP control group, LBP mat exercise group, and LBP I-Zer exercise group in 23-25 aged men. Visual analogue scale (VAS) and electromyography (EMG) were used to evaluate the degree of pain and the muscle activity in LBP patients. Root mean square (RMS), median frequency (MDF), and mean frequency (MNF) were checked by EMG power spectrum analysis on longissimus thoracic (LT), iliocostalis lumborum (IL), mulitifidus (M), and rectus abdominis (RA). LBP mat exercise program and LBP I-Zer exercise program were conducted 5 sets once time, 3 times per week during 6 weeks. The two-way ANOVA with repeated measure was used to check the pain degree and muscle activity. The present results showed that muscle activity in the LBP I-Zer exercise group was increased compared to the LBP mat exercise group and LBP control group $(P<0.05)$. LBP I-Zer exercise group and LBP mat exercise group showed increased mean frequency in $L T, I L, M$, and $R A$ muscles than the LBP control group. Therefore, LBP patients performed isometric exercise may have positive effect to reduce pain degree and to increase muscle activity. Especially, LBP I-Zer exercise type showed more effectiveness in reducing pain degree and enhancing muscle activity.

Keywords: Isometric exercise, Traction, Muscle activity, Low back pain, I-Zer

\section{INTRODUCTION}

The $80 \%$ of the population suffer from low back pain (LBP) at least once or twice in a life time. LBP is known to be induced by tissue damage, muscle weakness, and psychological factor, etc (Mayer et al., 2012). Most of patients with LBP show weakened muscle strength in lumbar spine area (Solomonow et al., 2012). Increasing strength of lumbar muscle is the most important therapeutic modality for the patient with LBP. Isotonic, isokinetic, and isometric exercise has been used to improve muscle strength and endurance of muscles in lumbosacral area. Isometric exercise is safe from injury and has potent efficacy for increasing muscle strength, and it can apply to the patients with motion limitation (Kurt and Pekünlü, 2015). Mat exercise is the most used type of isometric exercise, however this exercise has difficult in controlling muscle power (Kim, 2005). The majority of isometric and resistance exercise program used the Medex device. Medex device showed improvement in pain relief and increment in muscle activity of the lumbar muscle (Hong et al., 2010). Medex device isotonic contraction was accompanied by an extension of the lumbar spine. In the present study, Medex device was to use as a device for I-Zer isometric contraction of the lumbar spine. The device also has the effect on intermittent traction with isometric contraction.

Traction therapy relaxes ligaments and muscles around the spine. And it reduces the load of the pressure within the disc. Traction techniques for patients with LBP reduce pressure on the nerve roots. Traction therapy has been used for reducing pain in the LBP (Diab and Moustafa, 2013; Wegner et al., 2013).
${ }^{*}$ Corresponding author: Hye-Sang Park

Department of Sports Health Management, University of Suwon, 17 Wauan-gil, Hwaseong 18323, Korea

Tel: +82-31-229-8103, Fax: +82-31-229-2563, E-mail: wini-phs@hanmail.net Received: July12, 2015 / Accepted: August 17, 2015
This is an Open Access article distributed under the terms of the Creative Commons Attribution Non-Commercial License (http://creativecommons.org/licenses/by-nc/3.0/) which permits unrestricted non-commercial use, distribution, and reproduction in any medium, provided the original work is properly cited. 
I-Zer exercise device is developed for isometric exercise, and this device induces intermittent movement in the spinal vertebrae by traction, and results in correction of alignment in the balance of the left and right muscles. This device has wide been used clinically, however the exact efficacy has not been proved. In this study, we compared the effects of mat exercise and I-Zer exercise device on pain level and muscle activity in the patients with LBP.

\section{MATERIALS AND METHODS}

\section{Participants}

In this study, men age 23-25 were assigned into LBP control group, LBP mat exercise group, and LBP I-Zer exercise group ( $\mathrm{n}=$ 20 in each group). All participants were proved by doctor's examination to perform exercise. There were no statistically meaningful differences for variables (Table 1).

\section{Experimental procedures}

\section{Pain scale test}

Visual analogue scale (VAS) can be easily measured by a subjective pain score sheets, it is wildly used for the determination of the LBP. In this study, VAS is divided into vertical lines with every $10 \mathrm{~mm}$, from 0 point to 5 points.

\section{Muscle activity test}

Each study representative of the spectrum was used for the determination of differences in values that indicate the lumbar muscle of LBP. Values are different from normal sort of electromyography (EMG: MES9000, Apsun Inc., WA, USA), so EMG data were included in this study

EMG measures muscle activity and then skin was wiped using alcohol. And EMG data were collected using the MES 9000. The electrodes were attached both sides on LT $(3 \mathrm{~cm}$ lateral to the first lumbar spinous process), IL ( $5 \mathrm{~cm}$ lateral to the second lumbar spinous process), $\mathrm{MF}$ ( $3 \mathrm{~cm}$ lateral to the fifth lumbar spinous process), and upper and lower portion in rectus abdominis (RA). The reference electrode was attached on 5th lumbar spine. For the determination of maximal voluntary isometric contraction (MVIC) in lumbar muscles, trunk was extended. For the MVIC in abdominal muscle, trunk was flexed. MVIC was determined 3 times during 5 sec with 2 min interval (MES 900 manual).

\section{Lumbosacral isometric exercise program}

Mat exercise and I-Zer exercise (101, 4CU) was conducted 5 set once time, three times per week and continued six weeks. Lumbosacral exercise on the mat was carried out in the same movement as I-Zer. The mat exercise has $5 \mathrm{sec}$ exercise time and $5 \mathrm{sec}$ rest time (Table 2).

\section{Statistical analyses}

Significant difference in EMG measurement items and VAS pain level was analyzed by two-way ANOVA with repeated measure using statistical program SPSS (Ver. 21). $P<0.05$ was considered as significant.

\section{RESULTS}

\section{Changes in pain scale}

VAS pain rating index was decreased by mat exercise and I-Zer exercise (Fig. 1). I-Zer exercise showed more potent effect on pain relief compared to the mat exercise $(P<0.05)$.

Table 1. Characteristics of participants $(M \pm S D)$

\begin{tabular}{lccccc}
\hline & $n$ & Age $(\mathrm{yr})$ & Height $(\mathrm{cm})$ & Weight $(\mathrm{kg})$ & $\mathrm{BMl}\left(\mathrm{kg} / \mathrm{m}^{2}\right)$ \\
\hline LBP control & 20 & $20.50 \pm 2.36$ & $173.23 \pm 3.34$ & $70.93 \pm 8.06$ & $22.69 \pm 1.75$ \\
LBP Mat exercise & 20 & $21.75 \pm 1.08$ & $174.55 \pm 4.66$ & $68.83 \pm 8.73$ & $21.56 \pm 2.65$ \\
LBP I-Zer exercise & 20 & $21.63 \pm 0.88$ & $174.60 \pm 5.61$ & $69.49 \pm 7.32$ & $21.52 \pm 2.33$
\end{tabular}

LBP, low back pain patients; BMI, Body mass index.

Table 2. Isometric exercise program

\begin{tabular}{|c|c|c|c|c|}
\hline & Type & Frequency & Intensity (RPE) & Time \\
\hline LBP Mat exercise & Isometric exercises & 5 set, 3 times/week & 'Light' or 'little difficult' & $\begin{array}{c}\text { Warming-up stretching: } 3 \text { min } \\
\text { exercise } 5 \mathrm{sec} / \text { rest } 5 \mathrm{sec}\end{array}$ \\
\hline LBPI-Zer exercise & Isometric exercises + traction & For 6 week & & Cool-down stretching: 3 min \\
\hline
\end{tabular}

LBP, low back pain patients; RPE, rating of perceived exertion 
Rhyu HS, et al. - The effects of isometric exercise on low back pain

Table 3. Changes in muscle activity before and after six weeks of isometric exercise $(M \pm S D, m V)$

\begin{tabular}{|c|c|c|c|c|c|c|c|}
\hline \multirow{2}{*}{ Muscle } & \multirow{2}{*}{ Item } & \multicolumn{2}{|c|}{ LBP Control } & \multicolumn{2}{|c|}{ LBP Mat exercise } & \multicolumn{2}{|c|}{ LBP I-Zer exercise } \\
\hline & & Pre & Post & Pre & Post & Pre & Post \\
\hline Longissimus thoracic (LT) & RMS & $250.00 \pm 2.78$ & $252.13 \pm 0.92$ & $253.40 \pm 3.11$ & $262.15 \pm 4.43^{*}$ & $242.45 \pm 4.55$ & $274.70 \pm 8.38^{*}$ \\
\hline Longissimus thoracic (LT) & MDF & $33.40 \pm 2.91$ & $32.30 \pm 8.21$ & $34.90 \pm 1.97$ & $44.10 \pm 1.77^{*}$ & $34.30 \pm 1.71$ & $45.80 \pm 2.28^{*}$ \\
\hline Longissimus thoracic (LT) & MNF & $45.60 \pm 2.06$ & $47.21 \pm 6.06$ & $44.05 \pm 1.90$ & $54.90 \pm 1.55$ & $44.60 \pm 1.81$ & $57.50 \pm 2.60^{*}$ \\
\hline lliocostalis lumborum (IL) & RMS & $260.40 \pm 1.95$ & $262.14 \pm 2.29$ & $259.85 \pm 2.79$ & $272.00 \pm 3.30^{*}$ & $245.55 \pm 5.41$ & $275.80 \pm 5.78^{*}$ \\
\hline Iliocostalis lumborum (IL) & MDF & $38.80 \pm 4.96$ & $39.10 \pm 2.93$ & $38.65 \pm 1.66$ & $47.10 \pm 1.86^{*}$ & $38.40 \pm 1.75$ & $48.80 \pm 2.46^{*}$ \\
\hline lliocostalis lumborum (IL) & MNF & $48.80 \pm 1.93$ & $47.62 \pm 2.23$ & $45.80 \pm 1.85$ & $51.40 \pm 1.59$ & $46.6 \pm 1.78$ & $61.35 \pm 3.09^{*}$ \\
\hline Multifidus (MF) & RMS & $239.5 \pm 5.87$ & $240.5 \pm 3.12$ & $236.00 \pm 5.69$ & $250.25 \pm 6.10^{*}$ & $236.35 \pm 5.83$ & $256.95 \pm 5.91^{*}$ \\
\hline Multifidus (MF) & MDF & $28.40 \pm 3.89$ & $27.30 \pm 2.91$ & $28.7 \pm 4.75$ & $32.55 \pm 5.69$ & $28.5 \pm 3.81$ & $36.80 \pm 3.83^{*}$ \\
\hline Multifidus (MF) & MNF & $37.80 \pm 3.76$ & $38.79 \pm 1.06$ & $38.3 \pm 3.01$ & $42.65 \pm 2.77$ & $38.9 \pm 2.07$ & $48.15 \pm 2.99^{*}$ \\
\hline Rectus abdominis (RA) & RMS & $255.00 \pm 2.78$ & $257.00 \pm 1.42$ & $253.40 \pm 3.11$ & $272.15 \pm 4.43^{*}$ & $242.45 \pm 4.55$ & $265.50 \pm 5.01^{*}$ \\
\hline Rectus Abdominis (RA) & MDF & $32.40 \pm 2.91$ & $31.64 \pm 2.21$ & $32.75 \pm 2.76$ & $35.75 \pm 2.91$ & $33.60 \pm 3.57$ & $43.90 \pm 3.87^{*}$ \\
\hline Rectus Abdominis (RA) & MNF & $41.60 \pm 2.06$ & $42.56 \pm 3.07$ & $40.10 \pm 3.72$ & $45.75 \pm 3.71$ & $41.15 \pm 3.86$ & $52.90 \pm 5.24^{*}$ \\
\hline
\end{tabular}

$\mathrm{LBP}$, low back pain patients; $\mathrm{RMS}$, root mean square; $\mathrm{MDF}$, median frequency; $M N F$, mean frequency. ${ }^{*} P<0.05$.

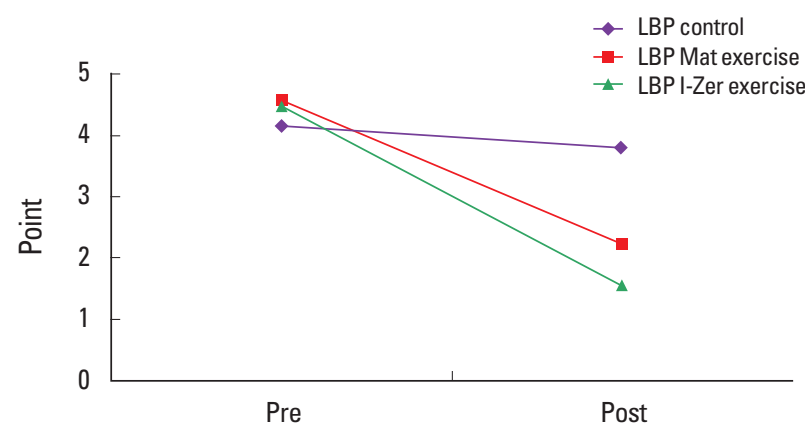

Fig. 1. The change in pain levels using VAS.

\section{Changes in the muscle activity}

Muscle activity was increased by mat exercise $(P<0.05)$ and I-Zer exercise $(P<0.05)$ (Table 3). I-Zer exercise showed more potent effect on muscle activation compared to the mat exercise $(P<$ $0.05)$.

\section{DISCUSSION}

Many studies have been made to identify the cause and treatment of LBP. The experience back pain avoids physical activity to decrease pain, which induces muscular atrophy. For the LBP persons, exercise program reducing lumbar pain has been recommended (Grgić, 2014). However, exercise therapy for pain control has not widely been used clinically. Lumbar rehabilitation exercise program increased erector spinae muscle size and reduced a pain and disability (Lee et al., 2012). Movement in lumbar rehabilitation program on the mat includes pilates, stretching, and gym, etc. Pilates on the mat enhanced muscle activity and reduced low back pain (Menacho et al., 2010). Traction therapy also reduced chronic LBP (van Middelkoop et al., 2011).

In this study, we used isometric exercise, mat exercise and I-Zer exercise, and I-Zer exercise showed more effectiveness on pain relief and muscle activation by decreasing traction and inducing relaxation. Generally, isometric training therapy (mat exercise) and exercise using a lumbosacral I-Zer device (I-Zer exercise) are designed to reduce pain by traction. Conservative treatment includes isometrics training on mat and I-Zer exercise. These exercise programs show effectiveness on reducing pain and improving function in musculoskeletal diseases (Choi et al., 2005; Taylor et al., 2007).

Increment of muscle strength and balance in lumbar spine and relief of pain could be achieved by stabilizing exercise (Han et al., 2010; Park et al., 2012), functional exercise (Shin et al., 2011), resistance exercise (Ju, 2006), and rehabilitation exercise (Lee, 2008). In the present study, disability in the activity of daily living (ADL) was decreased by isometric training on mat exercise and I-Zer exercise. These exercises showed effectiveness on LBP patients. This study will be helpful to the clinical practice. The present results could be used as the underlying method to develop more advanced techniques reducing LBP.

\section{CONFLICT OF INTEREST}

No potential conflict of interest relevant to this article was reported. 


\section{REFERENCES}

Choi G, Raiturker PP, Kim MJ, Jin CD, Chae YS, Lee SH. The effect of early isolated lumbar extension exercise program for patients with herniated disc undergoing lumbar discectomy. Neurosurgery 2005;57:762772 .

Diab AA, Moustafa IM. The efficacy of lumbar extension traction for sagittal alignment in mechanical low back pain: a randomized trial. J Back Musculoskelet Rehabil 2013;26:213-220.

Grgić V. Exercise program for chronic low back pain based on common clinical characteristics of patients. Lijec Vjesn 2014;136:156-166.

Han JE, Kim JH, Lee WJ, Kim KT, Hwang HS. Effects of body balance and trunk temperature on modes of waist-hip exercise stability after operated by percutaneous endoscopic discectomy. Korean J Sports Sci 2010; 19:1173-1187.

Hong SL, Kang KH, Kim TK, Lee JS, Yoon SJ. Effects of lumbar stabilization exercise with rehabilitative ultrasound imaging on lumbar deep muscle activity and lumbar isometric strength in athletes with chronic low back pain. J Sport Leisure Stud 2010;40:621-634.

Ju SB. The effects of medex lumbar extension program and progressive resistance exercise program on lumbar extension muscle strength, visual analogue scale in patients of spondylolisthesis. Korean Society Sports Sci 2006;15:673-681.

Kim TY. The effects of spinal stability exercise using the sling and mat. Korean Sports Research 2005;16:273-280.

Kurt C, Pekünlü E. Acute effect of whole body vibration on isometric strength, squat jump, and flexibility in well-trained combat athletes. Bio Sport 2015;32:115-122.

Lee DJ, Lee HJ, Han SW. The Influence of a exercise program on muscle thickness, maximum muscular strength, and pain reduction in the trunk region of participants with low back pain. J Sport Leisure Stud

\section{2;49:811-819.}

Lee JG. Effect of the rehabilitation exercise program on lumbar extension strength and physical self-concept in patients suffering lumbar disc herniations. J Sport 2008;32:859-867.

Mayer TG, Neblett R, Cohen H, Howard KJ, Choi YH, Williams MJ, Perez Y, Gatchel RJ. The development and psychometric validation of the central sensitization inventory. Pain Pract 2012;12:276-285.

Menacho MO, Obara K, Conceição JS, Chitolina ML, Krantz DR, da Silva RA, Cardoso JR. Electromyographic effect of mat pilates exercise on the back muscle activity of healthy adult females. J Manipulative Physiol Ther 2010;33:672-678.

Park JK, Kim KW. The analysis of stabilization exercise on lumbar extension strength, balance ability in adult female of chronic back pain patients. Korean J Sports Sci 2012;21:1129-1138.

$\mathrm{Shin} \mathrm{CH}$, Jeon KG. The effects of functional exercise program on lumbar extension strength and stability in middle-aged women of degenerative disc patients. J Physical Growth Motor Development 2011;19:291296.

Solomonow M, Zhou BH, Lu Y, King KB. Acute repetitive lumbar syndrome: a multi-component insight into the disorder. J Bodyw Mov Ther 2012;16:134-147.

Taylor NF, Dodd KJ, Shields N, Bruder A. Therapeutic exercise in physiotherapy practice is beneficial : a summary of systematic reviews 20022005. Australian J Physiother 2007;53:7-16.

van Middelkoop M, Rubinstein SM, Kuijpers T, Verhagen AP, Ostelo R, Koes BW, van Tulder MW. A systematic review on the effectiveness of physical and rehabilitation interventions for chronic non-specific low back pain. Eur Spine J 2011;20:19-39.

Wegner I, Widyahening IS, van Tulder MW, Blomberg SE, de Vet HC, Brønfort G, Bouter LM, van der Heijden GJ. Traction for low-back pain with or without sciatica. Cochrane Database Syst Rev 2013;8:CD003010. 